from 8 mos to 10 yrs. - Gross behavior disorder was a major handicap, schooling was interrupted, and intelligence deteriorated in the years before operation. EEG was of limited value, both hemispheres often being involved. High-voltage discharges occurred in the preserved hemisphere. The median age at operation was $11 \frac{1}{2}$ yrs (7-17 yrs). Follow-up ranged from 1-36 yrs.

Post-operatively, (1) habitual epilepsy was interrupted with complete freedom in 11 (65\%); (2) behavior improved, sometimes dramatically; (3) drop in IQ was halted; (4) the residual homonymous hemianopia was not a major handicap; and (5) one patient developed hemosiderosis of the postoperative cavity and died 11 years after operation.

The authors make the following recommendations: (1) establish that the lesion is unilateral and medication has been tried fully, (2) weigh dangers of status epilepticus, escalating behavior disorder, and deterioration of IQ when operation is delayed compared to benefits and low risks of early surgery; (3) use improved operative techniques with dissection of cortex in single piece (Falconer and Rushworth) and reconstruction and closure of cavity (Adams), and (4) regular follow-up with CT scans. (Lindsay J, Ounsted C, Richards P. Hemispherectomy for childhood epilepsy: A 36-year study. Dev Med Child Neurol $1987 ; 29: 592-600)$.

COMMINT. These authors have found hemispherectomy to be of considerable benefit to children with hemiplegic epilepsy and its concomitant social, psychological and medical disabilities. The surgical treatment of intractable epilepsy offers an alternative to chronic anticonvulsant drugs with their attendant adverse effects. With improved techniques now available the surgical approach should be considered more frequently in preference to a dogged persistence and reliance on medicines alone. (ped Neur Briefs 1987;1:24).

\title{
INFANTILE SPASMS AND ACTH DOSAGE
}

Results of corticotropin treatment of 33 patients with infantile spasms are reported from the Instituto Clinica Pediatrica, Universita di Siena, Italy. The etiology was undetermined in 8 and secondary in 25 (pre- or perinatal distress in 17 and tuberous sclerosis in 2). Pyridoxine $300 \mathrm{mg}$ IV tried in all cases initially with EEG monitoring was without effect. ACTH 2 units $/ \mathrm{kg}$ daily for 10 days followed by alternate day treatment for 10 days with a repeat course in some resulted in improvement in all idiopathic cases (complete in 6) and in 15 secondary cases (complete only in 2). The authors advocate low doses and short courses of ACIM, claiming results comparable with larger amounts (up to 10-12 u/kg/day) for longer periods ( $3 \mathrm{mos}$ ) employed by some. (Fois A et al. Further observations on the treatment of infantile spasms with corticotropin. Brain Dev 1987; $\underline{9}: 82-84$. Ibid, Eur J Pediatr 1983; $42: 51$.

COMMENT. The mechanism of the anticonvulsant action of ACTH in infantile spasms is unknown. It is probably independent of the adrenal (Neurology 1965;15:1136) and a possible direct CNS effect is a reason proposed for the use of high dosage schedules. In agreement with the present study, my own results with smaller doses, 10-20 units daily irrespective of body weight for 20 days, have equalled those reported with higher doses and longer courses, and serious side effects have been avoided. 